\title{
Prevalência e distribuição espacial da leishmaniose visceral em cães do município de Juatuba, Minas Gerais, Brasil
}

\author{
Prevalence and spatial distribution of visceral leishmaniasis in dogs Juatuba, Minas Gerais, Brazil
}

\author{
Luiz Felipe Nunes Menezes Borges ${ }^{\mathrm{I}, \mathrm{II}}$ Eliane Gonçalves Paiva Lopes $^{\mathrm{I}}$ \\ Ana Cláudia Parreiras de Freitas ${ }^{\mathrm{I}}$ Marcos Xavier Silva ${ }^{\mathrm{I}}$ João Paulo Amaral Haddad \\ José Ailton da Silva ${ }^{\mathrm{I}}$ Rafael Romero Nicolino ${ }^{\mathrm{I}}$ Danielle Ferreira de Magalhães Soares ${ }^{\mathrm{I}^{*}}$
}

RESUMO

\begin{abstract}
O presente estudo teve como objetivo determinar a prevalência e a distribuição espacial da leishmaniose visceral em cães (LVC) do município de Juatuba, Minas Gerais, Brasil, no ano de 2010. Foi realizado um estudo observacional transversal por meio de coleta de amostras sanguíneas de 957 cães, definidos aleatoriamente em todos os bairros do município. No diagnóstico sorológico, foram utilizados os testes de Imunofluorescência Indireta (IFI) e Teste deAdsorção Enzimática (ELISA), preconizados pelo Ministério da Saúde (MS). Foram marcadas as coordenadas geográficas dos imóveis participantes da pesquisa para verificar a distribuição espacial dos casos caninos. A prevalência da LVC foi estimada em 10,6\%, com variação de 3 a 50\%, distribuída em $70,6 \%$ dos bairros do município. A distribuição espacial pode ser observada por meio da varredura de agrupamentos e obtida à demarcação das áreas de risco diferenciado perante a ocorrência da doença no município de Juatuba. Foi observado aumento de 2,80 vezes mais chances em adquirir a LVC no cluster primário. A partir deste trabalho, as ações de prevenção e controle à LVC foram feitas de acordo com a especificidade de cada localidade, para evitar a expansão da doença entre os cães e novos casos humanos em Juatuba, Minas Gerais.
\end{abstract}

Palavras-chave: Leishmania infantum, georreferenciamento, indicador epidemiológico.

\section{ABSTRACT}

The objective of the present study was to determine the prevalence and spatial distribution of visceral leishmaniasis in dogs (VLD) at Juatuba city, Minas Gerais, Brazil, in 2010. A crosssectional observational study was performed collecting blood samples from 957 dogs, defined randomly in all city districts. For the sorological diagnosis was used indirect immunofluorescence (IIF) and enzyme-linked immunosorbent assay (ELISA), recommended by the Ministry of Health. Geographical coordinates of properties that participated in the survey were marked in order to check the spatial distribution of canine cases. The VLD prevalence was estimated in $10.6 \%$, ranging from $3 \%$ to $50.0 \%$, distributed between $70.6 \%$ of city districts. Spatial distribution could be observed by scanning the clusters and according to the disease occurrences the demarcation of different risk areas could be obtained. There was an increase of 2.80 times more likely to acquire the $V L D$ on the primary cluster. As of this research the prevention and control actions for VLD were made according to the specificity of each location to prevent the disease spread among dogs and new human cases.

Key words: Leishmania infantum, georeferencing, epidemiological indicator.

\section{INTRODUÇÃO}

A leishmaniose visceral (LV) é uma doença causada por protozoários do gênero Leishmania e a espécie incriminada no Brasil, pela forma visceral, é a L. chagasi, atualmente, considerada sinonímia da L. infantum (ROMERO e BOELART, 2010). A Lutzomyia (Lutzomyia) longipalpis é a principal espécie transmissora da $\mathbf{L}$. infantum no Brasil. O cão (Canis familiaris) é uma importante fonte de infecção para o vetor em ambiente urbano e geralmente está associado com a ocorrência de casos humanos (OLIVEIRA et al., 2001; ARAÚJO, 2011). É considerada como uma zoonose com impacto na Saúde Pública, pois envolve fatores de risco de ordem ambientais, socioeconômicos, sanitários, políticos e educacionais.

'Departamento de Medicina Veterinária Preventiva, Área de Epidemiologia, Escola de Veterinária, Universidade Federal de Minas Gerais (UFMG), Av. Antônio Carlos, 6627, B. São Francisco, CP 567, 30123-970, Belo Horizonte, MG, Brasil. E-mail: daniellef@ufmg.br. *Autor para correspondência.

"Serviço de Controle de Zoonoses, Vigilância em Sáude, Secretaria Municipal de Saúde, Prefeitura Municipal de Juatuba, Juatuba, MG, Brasil. Recebido 17.06.13 Aprovado 06.09.13 Devolvido pelo autor 29.11.13 CR-2013-0829.R1 
Possui ampla distribuição mundial e registro de casos em quase todos os continentes. No Brasil, configura-se como uma endemia em franca expansão geográfica, atingindo as cinco regiões brasileiras (BRASIL, 2011). O controle da doença deve ser feito de maneira estratégica, mediante a identificação e estratificação das áreas de risco pelos inquéritos sorológicos caninos e entomológicos, investigação epidemiológica dos casos humanos e condições ambientais adversas (BRASIL, 2006).

O município de Juatuba está situado a $50 \mathrm{~km}$ da capital mineira, Belo Horizonte. A região é delimitada por extensa área de campos, cerrado e matas. O primeiro caso de LV em humanos foi registrado em 1999 e posteriormente ocorreram mais cinco casos, distribuídos nos anos de 2007, 2011 (três casos) e 2012. Juatuba foi caracterizada como uma área de transmissão esporádica, de acordo com a classificação de áreas de transmissão para LV do MS. É necessária a realização de investigação epidemiológica no município para conhecimento da distribuição da doença e planejamento das ações de prevenção e controle adequados a cada localidade (BRASIL, 2006).

$\mathrm{O}$ presente estudo objetivou determinar a prevalência e a distribuição espacial da leishmaniose visceral canina (LVC) no município de Juatuba, Minas Gerais, Brasil, no ano de 2010, para assim determinar ações específicas para vigilância e controle da LV no município.

\section{MATERIAL E MÉTODOS}

Foi realizado um estudo epidemiológico observacional do tipo transversal, no período de abril a outubro de 2010, para determinação da prevalência e distribuição espacial da LVC no município de Juatuba, integrante da Região Metropolitana de Belo Horizonte (RMBH), Minas Gerais, Brasil.

O cálculo amostral canino foi feito pela ferramenta Statcalc, para pesquisa de populações do programa EpiInfo, versão 6.04. Calculou-se a amostra considerando população finita (nível de confiança de $95 \%$, precisão de $20 \%$ e prevalência esperada para LV de 8\%), totalizando 1.005 cães.

A seleção dos quarteirões a serem trabalhados em cada bairro foi feita por sorteio por amostragem aleatória simples. Já o imóvel a ser amostrado e o cão escolhido para coleta sanguínea foram definidos pela amostragem aleatória sistemática (MEDRONHO et al., 2006), padronizando o $3^{\circ}$ imóvel à direita do início do quarteirão, e o cão cujo nome fosse o $3^{\circ} \mathrm{em}$ ordem alfabética em residência com mais de um animal.
Foram coletados aproximadamente $3 \mathrm{~mL}$ de sangue de cada cão por meio de venopunção cefálica ou jugular, armazenados em caixa térmica para posterior centrifugação a $3.000 \mathrm{rpm}$ por 5 minutos e encaminhados ao Laboratório de Leishmaniose da Escola de Veterinária da Universidade Federal de Minas Gerais (UFMG). Para o diagnóstico da LVC, foram utilizados os testes de Imunofluorescência Indireta (IFI), kit BioManguinhos ${ }^{\circledR} /$ Fundação Oswaldo Cruz (FIOCRUZ) e Teste de Adsorção Enzimática (ELISA), Kit ELISA $/ S 7^{\circledR}$ da Biogene Indústria e Comércio Ltda, preconizados pelo Ministério da Saúde (MS), com análises em paralelo e conforme bula de cada fabricante. A amostra do cão foi considerada na IFI sororreagente para títulos $\geq 40$ e indeterminada para reação inconclusiva, ou seja, fluorescência de membrana não é completa, independentemente do resultado de ELISA.

Para análise e representação espacial dos casos de LVC em Juatuba, foram marcadas as coordenadas geográficas, obtendo-se a localização de cada imóvel participante. Para a verificação das áreas do município de Juatuba em que o número de casos caninos da LV encontrava-se acima do esperado, foi utilizado um método de estatística espacial de varredura, para detecção de clusters, denominado Purely Spatial Multinomial, realizada com auxílio do software SatScan. Para a confecção dos mapas e visualização espacial dos casos caninos de LV do município, utilizaram-se os recursos do aplicativo de mapeamento MapInfo, versão 9.5.

\section{RESULTADOS E DISCUSSÃO}

Foram realizadas coletas de sangue em 957 cães, distribuídos proporcionalmente ao número de cães e aleatoriamente em todos os bairros em que se realizava o Programa Nacional de Controle da Dengue, de acordo com os dados do Serviço de Controle de Zoonoses de Juatuba.

A prevalência para LVC no município foi de 10,6\% (IC 95\%: 10,07-11,13) com 102 amostras reagentes; 805 não reagentes e $50 \mathrm{com}$ sorologia indeterminada. A prevalência da LVC variou de 3 a $50 \%$ (Tabela 1). Em 70,6\% dos bairros do município foram detectados cães com sorologia reativa, $47 \%$ com sorologia indeterminada e $85,3 \%$ com ambos os resultados.

A elevada soroprevalência encontrada para a LVC em Juatuba pode estar relacionada à atual situação de expansão do ambiente urbano, que vem ocorrendo no município, como observado nos estudos de LUZ et al. (2001) e DINIZ et al. (2008). Associada 
Tabela 1 - Distribuição da taxa de prevalência da leishmaniose visceral canina dividida por estratos dos bairros de Juatuba, Minas Gerais, Brasil, 2010.

\begin{tabular}{|c|c|c|c|}
\hline Prevalência por estratos & Cães examinados & Bairros & Características gerais \\
\hline $0 \%$ & 151 & $\begin{array}{l}\text { Boa Vista, Carioca, Castelo Branco, } \\
\text { Coqueiro Verde, Dona Francisca, Distrito } \\
\text { Industrial II, Parque Alvorada, Sol } \\
\text { Nascente, CondomínioVillage, Veredas } \\
\text { da Serra I. }\end{array}$ & $\begin{array}{l}\text { Bairros menores, menor número de } \\
\text { moradores, deficiência de saneamento } \\
\text { básico, ruas não pavimentadas, população } \\
\text { carente. }\end{array}$ \\
\hline 3 a $10 \%$ & 400 & $\begin{array}{l}\text { Canãa, Cidade Nova I e II, Diamantina, } \\
\text { Ilhéus, Jardim Boa Vista, Samambaia, } \\
\text { Santo Antônio, Serra Azul, Veredas da } \\
\text { Serra II, Vila Maria Regina II. }\end{array}$ & Aglomeração humana, população carente. \\
\hline 11 a $20 \%$ & 222 & $\begin{array}{l}\text { Bela Vista, Centro, Jardim Baviera, } \\
\text { Ponte Nova, Quinta da Boa Vista, São } \\
\text { Gerônimo, Vila Maria Regina I. }\end{array}$ & $\begin{array}{l}\text { Sítios, aglomeração canina, deficiência de } \\
\text { saneamento básico. }\end{array}$ \\
\hline Superior a $21 \%$ & 184 & $\begin{array}{l}\text { Cidade Nova III e IV, Eldorado, Satélite I } \\
\text { e II, Vila Verne. }\end{array}$ & $\begin{array}{l}\text { Aglomeração humana e canina, } \\
\text { deficiência de saneamento básico, ruas } \\
\text { não pavimentadas, população baixa } \\
\text { renda. }\end{array}$ \\
\hline
\end{tabular}

às alterações ambientais e condições inadequadas de moradia, devido à ocupação recente, podem contribuir também para a disseminação da LV na região.

Os resultados obtidos do inquérito sobre a LVC permitiram avaliar a sua distribuição no município de Juatuba. A utilização de mapa com a localização dos imóveis georreferenciados permite uma melhor visualização dos pontos referentes aos imóveis visitados, e áreas sem sinalização de pontos correspondem às regiões de matas, campos e cerrados (Figura 1). A prevalência detectada encontrase semelhante àquelas relatadas em estudos de amostragem aleatória no Brasil: CAMARGO-NEVES et al. (2001), em Araçatuba, São Paulo, com 12,1\% e variação de 4,1 a 25,8\%; FRANÇA-SILVA et al. (2003), em Montes Claros, Minas Gerais, com 9,7\%.

A soropositividade canina obtida distribuiu-se de forma heterogênea nas diversas localidades estudadas do município, podendo ser observadas quatro estratificações diferentes: ausência de casos de LVC; variação de 3 a 10\%; 11 a 20\%; e superior a $21 \%$ na prevalência. Essa diferença de variação entre as localidades pode ser explicada devido às características presentes nos diferentes bairros, tais como população de baixa renda, aglomeração canina e humana, presença de chácaras, deficiência de saneamento básico, lixo exposto na rua ou em locais inadequados e também devido à presença de animais dentro e ao redor do peridomicílio. Tais fatores podem contribuir para a manutenção da
LV em níveis elevados nessas localidades, e então explicar os maiores valores de soropositividade canina encontrados em Minas Gerais (MORENO et al., 2005; BORGES, 2011).

BORGES et al. (2009) verificaram que a presença de animais nas residências está associada ao risco de transmissão da leishmaniose visceral em humanos em Belo Horizonte. MORENO et al. (2005), em Minas Gerais, e MISSAWA \& LIMA (2006), no Mato Grosso, verificaram que o acúmulo de lixo e matéria orgânica no imóvel aumentou o risco de contrair a LV, por propiciarem um ambiente favorável à manutenção do vetor. A ação antrópica no processo de urbanização pode ter influenciado na determinação de ambientes associados à ocorrência dos casos de LV nos cães.

A realização de inquéritos sorológicos em cães, como neste trabalho, pode ser considerada de grande importância no controle do reservatório canino. A identificação dos casos serve de ferramenta para direcionar e priorizar ações específicas para cada localidade (BRASIL, 2006; ARAÚJO, 2011) e poderia auxiliar na detecção de focos silenciosos da doença. Por meio da varredura de agrupamentos, foi obtida a demarcação de áreas com risco diferenciado perante a ocorrência da doença no município de Juatuba (Figura 1; Tabela 2). Foram encontradas três áreas com riscos diferenciados, sendo classificadas como Cluster Primário, Cluster Secundário - $2 \mathrm{e}$ Cluster Secundário - 3. 


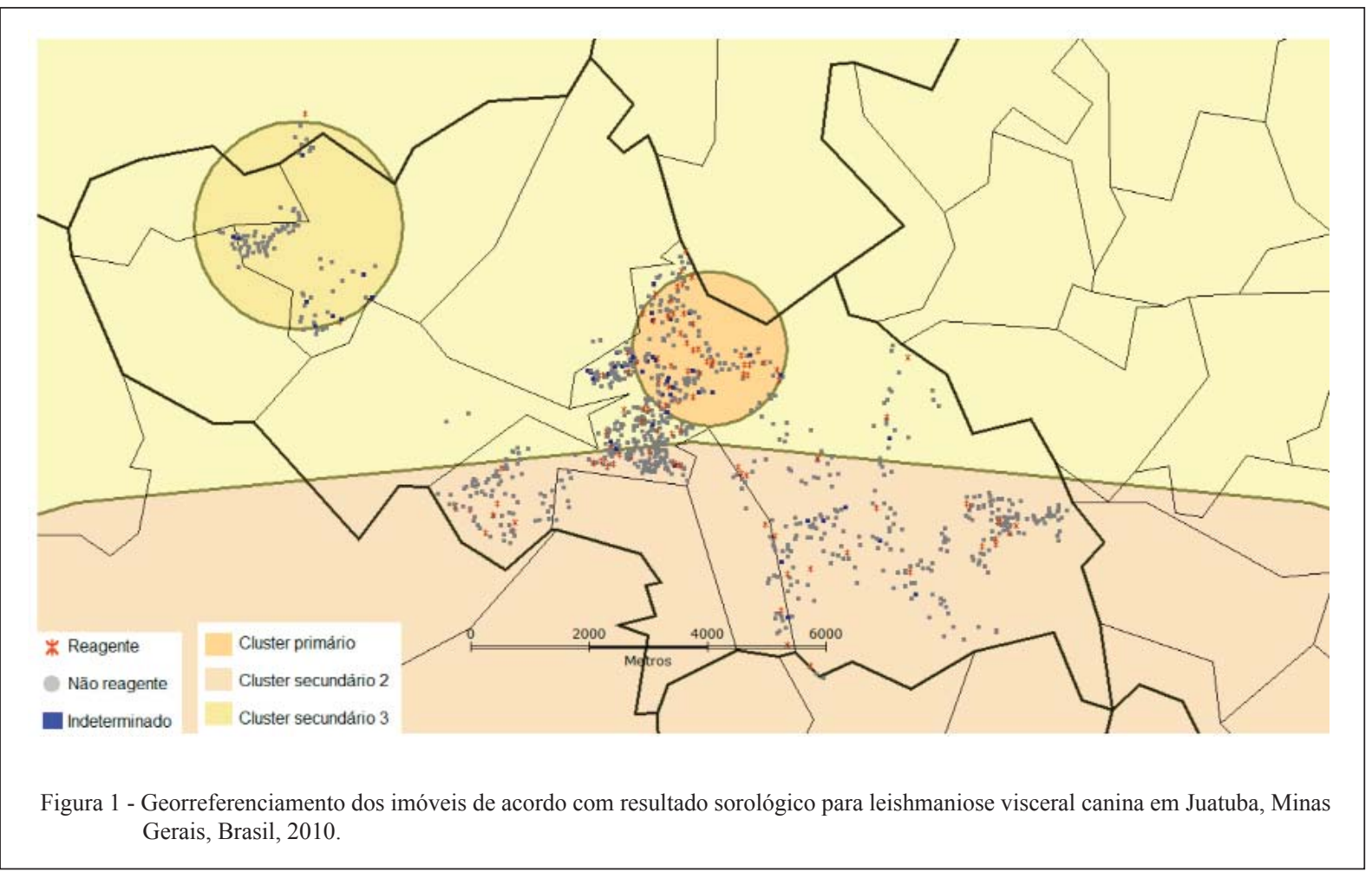

A distribuição de casos de LVC ocorreu de forma heterogênea em Juatuba, o que também foi verificado por CAMARGO-NEVES et al. (2001), em Araçatuba, São Paulo, e LOPES et al. (2010), em Belo Horizonte, Minas Gerais. Trabalhos que avaliam a distribuição espacial da LV são importantes para o direcionamento das ações de prevenção e controle adequadas. Além disso, o conhecimento dos casos de LVC pode servir de indicador para o surgimento de novos casos humanos, uma vez que a infecção no cão normalmente precede a doença no homem, como verificado por OLIVEIRA et al. (2001) em Belo Horizonte, Minas Gerais.

A medida de associação utilizada no cálculo dos clusters foi o risco relativo. No Cluster Primário, foi observado um risco de infecção canina de 2,80 vezes e 2,97 vezes em ser indeterminado para LVC (Tabela 2). Nessa área, estão presentes imóveis georreferenciados com prevalência canina encontrada com valor igual ou superior a $15 \%$. O risco de infecção para LVC pode ser pelo valor elevado da prevalência encontrado nos bairros presentes nesse agrupamento e as características socioambientais favoráveis à manutenção da doença.

Os Clusters Secundários - 2 e 3 não foram considerados como áreas de risco para ocorrência de LVC, pois, dentre os cães com resultado sorológico reativo para $\mathrm{LV}$, o número de casos esperados foi maior que os observados (Tabela 2). Adiferenciação do risco de ocorrência da doença pode ser influenciada pela forma de ocupação do espaço urbano (LOPES et al., 2010) como a encontrada nos diversos bairros de Juatuba.

Tabela 2 - Dados dos agrupamentos relacionados aos resultados sorológicos para leishmaniose visceral canina, Juatuba, Minas Gerais, Brasil, 2010.

\begin{tabular}{|c|c|c|c|c|c|c|c|c|c|c|}
\hline \multirow{2}{*}{ Cluster } & \multicolumn{3}{|c|}{ 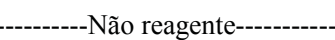 } & \multicolumn{3}{|c|}{-----------'Reagente------------ } & \multicolumn{3}{|c|}{---------Indeterminado--------- } & \multirow{2}{*}{ Valor de $\mathrm{P}$} \\
\hline & $\mathrm{O}$ & $\mathrm{E}$ & $\mathrm{RR}$ & $\mathrm{O}$ & $\mathrm{E}$ & $\mathrm{RR}$ & $\mathrm{O}$ & $\mathrm{E}$ & $\mathrm{RR}$ & \\
\hline Primário $(n=170)$ & 111 & 142,5 & 0,74 & 39 & 18,39 & 2,8 & 20 & 9,11 & 2,97 & 0,0001 \\
\hline Secundário $2(\mathrm{n}=476)$ & 421 & 399 & 1,12 & 46 & 51,5 & 0,81 & 9 & 25,5 & 0,21 & 0,0088 \\
\hline Secundário $3(\mathrm{n}=89)$ & 80 & 74,6 & 1,08 & 0 & 9,63 & 0 & 9 & 4,77 & 2,08 & 0,026 \\
\hline
\end{tabular}

$\mathrm{O}=$ valor observado $\mathrm{E}=$ Valor esperado $; \mathrm{e} \mathrm{RR}=$ risco relativo 
No Cluster Secundário 2, foi verificada maior probabilidade de o cão ser sorologicamente negativo (1,12 vez), provavelmente, pelo número menor de casos de LVC. O Cluster Secundário 3 abrange uma região mais afastada da área urbana do município, delimitado por uma extensa área verde e foi verificada maior probabilidade (2,08 vezes) do cão ser sorologicamente indeterminado para LV (Figura 1).

$\mathrm{O}$ georreferenciamento auxilia na visualização de áreas com maior risco de transmissão e permite a otimização de recursos humanos (CAMARGO-NEVES et al., 2001).

\section{CONCLUSÃO}

O município de Juatuba apresentou alta prevalência para LVC, com distribuição de forma heterogênea nas diversas localidades estudadas. Foram encontrados agrupamentos classificados de acordo com a ocorrência de cães sororeagentes para LV.

Os resultados deste trabalho permitirão direcionar as ações de prevenção e controle de maneira específica para cada localidade (de acordo com o risco), podendo assim priorizar as atividades em áreas com maior ocorrência da LVC. A realização de ações educativas, posteriores ao inquérito, a capacitação dos profissionais da área de saúde e palestras à população contribuirão significativamente para redução da expansão da doença entre os cães e possivelmente o impedimento da ocorrência de novos casos humanos em Juatuba.

\section{AGRADECIMENTOS}

Secretaria Municipal de Saúde de Juatuba e Secretaria de Estado de Saúde de Minas Gerais.

\section{COMITÊ DE ÉTICA E BIOSSEGURANÇA}

Comitê de Ética em Pesquisa (COEP), parecer número: ETIC 0044.0.203.000-10. Comitê de Ética em Experimentação Animal (CETEA), protocolo número: 018/10. Universidade Federal de Minas Gerais.

\section{REFERÊNCIAS}

ARAÚJO, V.E.M. Análise da distribuição espaço-temporal da leishmaniose visceral e perfil clínico-epidemiológico dos casos e óbitos, Belo Horizonte, Minas Gerais, 1994 a 2009.2011. 208f. Tese (Doutorado em Ciências) - Curso de Pós-graduação Parasitologia, do Instituto de Ciências Biológicas da Universidade Federal de Minas Gerais, MG.

BORGES, B.K.A. Fatores de risco associados ao perfil sorológico da Leishmaniose visceral em cães, Montes Claros/MG. 2011. 2011. 104f. Tese (Doutorado em Epidemiologia) - Curso de Pósgraduação em Ciência Animal da Escola de Veterinária, Universidade Federal de Minas Gerais, MG.
BORGES, B.K.A. et al. Presença de animais associada ao risco de transmissão da leishmaniose visceral em humanos em Belo Horizonte, Minas Gerais. Arquivo Brasileiro de Medicina Veteterinária e Zootecnia, Belo Horizonte, v.61, n.5, p. 1035-1043, 2009. Disponível em: $<$ http://www.scielo.br/scielo.php?script=sci arttext\&pid=S0102-09352009000500004>. Acesso em: 12 nov. 2012. doi:10.1590/S0102-09352009000500004.

BRASIL. Ministério da Saúde. Departamento de Vigilância Epidemiológica. Secretaria de Vigilância em Saúde. Manual de vigilância e controle da leishmaniose visceral. Secretaria de Vigilância em Saúde. Brasília: Ministério da Saúde, 2006. 120p. Disponível em: <http://portal.saude.gov.br/portal/arquivos/pdf/ manual_leish_visceral2006.pdf>. Acesso em: 22 jun. 2011.

BRASIL. Ministério da Saúde. Portal da Saúde - Glossário leishmaniose visceral. Secretaria de Vigilância em Saúde. Disponível em: <http://portal.saude.gov.br>. Acesso em: 15 jan. 2011.

CAMARGO-NEVES, V.L. et al. Utilização de ferramentas de análise espacial na vigilância epidemiológica de leishmaniose visceral americana - Araçatuba, São Paulo, Brasil, 1998-1999. Cadernos de Saúde Pública, v.17, n.5, p.1263-1267, 2001. Disponível em: $<$ http://www.scielo.br/scielo.php?script=sci arttext\&pid=S0102-311X2001000500026\&lng=en $>$. Acesso em: 22 dez. 2010. doi:10.1590/S0102-311X2001000500026.

DINIZ, S.A. et al. Animal reservoirs for visceral leishmaniasis in densely populated urban areas. Journal of Infection in Developing Countries, v.2, n.1, p.24-33, 2008. Disponível em: $<$ http://jidc.org/index.php/journal/article/view/19736384/178>. Acesso em: 22 nov. 2012.

FRANÇA-SILVA, J.C. et al. Epidemiology of canine visceral leishmaniasis in the endemic area of Montes Claros Municipality, Minas Gerais State, Brazil. Veterinary Parasitology, v.111, n.23, p.161-173, 2003. Disponível em: <http://www.sciencedirect. com/science/article/pii/S0304401702003515>. Acesso em: 12 jan.2011. doi: 10.1016/S0304-4017(02)00351-5.

LOPES, E.G.P. et al. Distribuição temporal e espacial da leishmaniose visceral em humanos e cães em Belo HorizonteMG, 1993 a 2007. Arquivo Brasileiro de Medicina Veterinária e Zootecnia, v.62, n.5, p.1062-1071, 2010. Disponível em: <http://www.scielo.br/scielo.php?pid=S010209352010000500007\&script $=$ sci arttext $>$. Acesso em: 15 fev. 2011. doi: 10.1590/S0102-09352010000500007.

LUZ, Z.M.P. et al. A urbanização das leishmanioses e a baixa resolutividade diagnóstica em municípios da Região Metropolitana de Belo Horizonte. Revista da Sociedade Brasileira de Medicina Tropical, v.34, p.249-254, 2001. Disponível em: $<$ http://www.scielo.br/scielo.php?script=sci arttext\& pid $=$ S0037-86822001000300004 $>$. Acesso em: 15 ago. 2012. doi: $10.1590 / \mathrm{S} 0037-86822001000300004$.

MEDRONHO, R.A. et al. Epidemiologia. In: TORRES, T.Z.G. Amostragem. São Paulo: Atheneu, 2006. Cap.20, p.283-294.

MISSAWA, N.A.; LIMA, G.B.M. Distribuição espacial de Lutzomyia longipalpis (Lutz \& Neiva, 1912) e Lutzomyia cruzi (Mangabeira, 1938) no Estado de Mato Grosso. Revista da Sociedade Brasileira de Medicina Tropical, v.39, n.4, p.337-340, 2006. Disponível em: $<$ http://www.scielo.br/scielo.php?script=sci arttext\&pid=S0037-86822006000400004>. Acesso em: 29 ago. 2012. doi: $10.1590 / \mathrm{S} 0037-86822006000400004$. 
MORENO, E.C. et al. Risk factors for Leishmania chagasi infection in an urban area of Minas Gerais State. Revista da Sociedade Brasileira de Medicina Tropical, v.38, n.6, p.456463, 2005. Disponível em: <http://www.scielo.br/scielo. php?script $=$ sciarttext $\quad$ \&pid $=$ S003786822005000600002\&lng=e n\&nrm=iso\&tlng=en>. Acesso em: 10 set. 2012. doi: 10.1590/ S0037-86822005000600002.

OLIVEIRA, C.L. et al. Spacial distribution of human and canine visceral leishmaniasis in Belo Horizonte, Minas Gerais State, Brazil,
1994-1997. Cadernos de Saúde Pública, v.7, p.1231-1239, 2001. Disponível em: <http://www.scielo.br/scielo.php?pid=S0102$311 \mathrm{X} 2001000500023 \&$ script $=$ sci arttext $>$. Acesso em: 07 jul. 2012. doi: 10.1590/S0102-311X2001000500023.

ROMERO, G.A.S; BOELAERT, M. Control of visceral leishmaniasis in Latin America - A systematic review. PLoS Neglected Tropical Disease, v.4, n.1, p.584, 2010. Disponível em: $<$ http://www.plosntds. org/article/info\%3Adoi\%2F10.1371\%2Fjournal.pntd.0000584>. Acesso em: 05 out. 2012. doi: 10.1371/journal.pntd.0000584. 